\title{
Answer to the Letter to the Editor of A. Agarwal et al. concerning "Rod fracture lengthening intervals in traditional growing rods: is there a relationship?" by P. Hosseini et al. Eur Spine J (2016); doi:10.1007/s00586-016-4786-8
}

\author{
Pooria Hosseini ${ }^{1}$
}

Received: 13 April 2017/Accepted: 14 April 2017/Published online: 19 April 2017

(c) Springer-Verlag Berlin Heidelberg 2017

We would like to thank the authors of this letter for their interest in our study [1]. To address their concerns, we would like to make it clear that the purpose of the current study was to determine whether there is a relationship between lengthening intervals and incidence of rod fracture in traditional growing rod (TGR) surgery. This study was not designed to validate previously reported finite element analysis (FEA) findings with clinical data. Therefore, the authors' comment, "The premise of their research was to test the conclusion of our previously published article(s), in a clinical scenario" is not correct.

They have commented that current study is not considering the magnitude of forces and, therefore, misunderstanding the FEA results. They also mentioned the ideal way to study shorter lengthening interval effects on rod fracture in a clinical setting is achievable through studying magnetically controlled growing rods (MCGR). In response, we feel it is important to outline the following points:

- Currently, the majority of experts recommend sixmonth distraction intervals of traditional growing rods (TGR). However, based on the clinical data only $24 \%$ of surgeons are following this recommendation [2], which strongly suggests the existence of a high degree of variation in lengthening intervals in TGR cases. In this study, we aimed to determine whether there is any correlation between lengthening intervals and rod fracture in TGR. In this context, the literature search for clinical evidence was inconclusive. For that reason,

Pooria Hosseini

phosseini@sandiegospinefoundation.org

1 San Diego Spine Foundation, 6190 Cornerstone Ct; Ste 212, San Diego, CA 92121, USA the current study gained the merit for investigation due to its novelty in the clinical field.

- Regarding the suggestion to consider "actual magnitude of forces" with different lengthening intervals, it is important to keep in mind that there are two basic types of risk factors for implant-related complications (IRC) in a clinical setting: modifiable and non-modifiable. Lengthening interval falls into the modifiable category that clinicians can control. On the other hand, the magnitude of exerted forces is non-modifiable in clinical setting. With currently available technology, the measurement of exerted forces on implanted rods is not possible. We are hopeful that future implant systems can address this shortfall.

- In line with the recommendation on studying MCGR, we are in agreement and we have proposed the study of correlation between lengthening intervals and rod fracture in MCGR in the last paragraph of the discussion section of the current article and a randomized trial of different lengthening intervals is underway.

\section{References}

1. Hosseini P, Pawelek JB, Nguyen S, Thompson GH, Shah SA, Flynn JM et al (2016) Rod fracture and lengthening intervals in traditional growing rods: is there a relationship? Eur Spine J. doi:10.1007/s00586-016-4786-8

2. Yang JS, McElroy MJ, Akbarnia BA, Salari P, Oliveira D, Thompson GH et al (2010) Growing rods for spinal deformity: characterizing consensus and variation in current use. J Pediatr Orthop 30(3):264-270 